\title{
EVALUACIÓN DE FERTILIZANTES AGRÍCOLAS EN LA PRODUCTIVIDAD DE LA MICROALGA Chlorella sorokiniana ${ }^{1}$
}

\author{
Ana Margarita Silva-Benavides ${ }^{2}$
}

\begin{abstract}
RESUMEN
Evaluación de fertilizantes agrícolas en la productividad de la microalga Chlorella sorokiniana. El objetivo del presente trabajo fue evaluar la productividad de la microalga verde Chlorella sorokiniana UTEX 1230 con fertilizantes foliares usados en la agricultura y la comparación con el medio nutritivo Kolwitz (K3). Las variables evaluadas fueron el crecimiento celular, la productividad, la concentración de clorofila, la fluorescencia $(\mathrm{Fv} / \mathrm{Fm})$, proteínas y carbohidratos. Se usaron dos fertilizantes comerciales con la composición química NPK 20-20-20 y NPK 22-10-7 como medios nutritivos. El experimento se realizó en el Instituto de Estudios para los Ecosistemas, Florencia, Italia durante el año 2014. Cada experimento se repitió cinco veces con tres réplicas cada uno, a una intensidad lumínica de $150 \mu \mathrm{mol}$ fotones $\mathrm{m}^{2} / \mathrm{s}$, temperatura de $28{ }^{\circ} \mathrm{C} \mathrm{y} \mathrm{CO}_{2} /$ aire (2:98 v/v). Con cada fertilizante se diseñaron los siguientes tratamientos: 1) NPK 20-20-20 (1,00 $\left.\mathrm{g} / \mathrm{l})+0,27 \mathrm{~g} / \mathrm{l} \mathrm{MgSO}{ }_{4} .7 \mathrm{H}_{2} \mathrm{O}, 2\right)$ NPK 22-10-7 (+2 MgO) $(1,00$ $\left.\mathrm{g} / \mathrm{l})+0,27 \mathrm{~g} / \mathrm{l} \mathrm{MgSO}{ }_{4} .7 \mathrm{H}_{2} \mathrm{O}, 3\right)$ NPK 22-10-7 (+2 MgO) $(1,00$ $\mathrm{g} / \mathrm{l}), 4)$ NPK 20-20-20 (1,00 g/l). Se utilizó el medio nutritivo Kolwitz (K3) como control. Las mayores concentraciones celulares, productividad, clorofila, fluorescencia, proteínas y carbohidratos, se cuantificaron en los medios provistos de sulfato de magnesio (NPK 20-20-20 (1,00 g/l) + 0,27 g/l $\mathrm{MgSO}_{4} \cdot 7 \mathrm{H}_{2} \mathrm{O}$, y NPK 22-10-7 (+2 MgO) $(1,00 \mathrm{~g} / \mathrm{l})+0,27 \mathrm{~g} / \mathrm{l}$ $\mathrm{MgSO}_{4} \cdot 7 \mathrm{H}_{2} \mathrm{O}$ ). Los menores crecimientos de la biomasa se observaron en los tratamientos carentes de este compuesto. El sulfuro y el magnesio tuvieron efecto positivo en el crecimiento y rendimiento del cultivo; así como las cantidades de urea y amonio en la composición química del fertilizante.
\end{abstract}

Palabras clave: fluorescencia de la clorofila, productividad microalgal, nitrógeno total, sulfato de magnesio.

\begin{abstract}
Evaluation of agricultural fertilizers on the productivity of microalgae Chlorella sorokiniana. The aim of this study was to evaluate the productivity of microalgae Chlorella sorokiniana UTEX 1230 with two different foliar fertilizers used in agriculture and the comparison with the nutrient medium Kolwitz (K3).Variables assessed were cell growth, productivity, chlorophyll concentration, fluorescence $(\mathrm{Fv} / \mathrm{Fm})$, proteins, and carbohydrates. Two commercial fertilizers with NPK 20-20-20 and NPK 22-10-7 formula were used as nutrient media. The study was conducted at the Institute of Ecosystem Studies, Florence, Italy, in 2014. Each experiment was repeated five times using three replicates in each experiment under a light intensity of $150 \mu \mathrm{mol}$ photons $\mathrm{m}^{2} / \mathrm{s}$, temperature $28{ }^{\circ} \mathrm{C}$ and $\mathrm{CO}_{2} /$ air $(2: 98 \mathrm{v} / \mathrm{v})$. The culture media were prepared by using the following fertilizers: 1 ) NPK 20-20-20 (1.00 g/l) + 0.27 g/l $\left.\mathrm{MgSO}_{4} .7 \mathrm{H}_{2} \mathrm{O}, 2\right) \mathrm{NPK}$ $\left.22-10-7(+2 \mathrm{MgO})(1.00 \mathrm{~g} / \mathrm{l})+0.27 \mathrm{~g} / 1 \mathrm{MgSO}_{4} .7 \mathrm{H}_{2} \mathrm{O}, 3\right)$ NPK 22-10-7 (+2 MgO) (1.00 g/l), 4) NPK 20-20-20 (1.00 $\mathrm{g} / \mathrm{l}$ ). The nutrient medium Kolwitz (K3) was used as a control. Higher cell concentrations, productivity, chlorophyll, fluorescence, proteins, and carbohydrates were obtained in cultures grown in the media prepared with magnesium sulfate (NPK 20-20-20 (1.00 g/l) + $0.27 \mathrm{~g} / \mathrm{l} \mathrm{MgSO}_{4} \cdot 7 \mathrm{H}_{2} \mathrm{O}$, and NPK 22-10-7 (+2 MgO) $\left.(1.00 \mathrm{~g} / \mathrm{l})+0.27 \mathrm{~g} / 1 \mathrm{MgSO}_{4} \cdot 7 \mathrm{H}_{2} \mathrm{O}\right)$. Lower biomass growth was obtained in cultures lacking magnesium sulfate. Sulfide and magnesium, as well as the amounts of urea and ammonia in the chemical composition of each fertilizer, had a positive effect on the culture growth and productivity.
\end{abstract}

Keywords: chlorophyll fluorescence, microalgal productivity, total nitrogen, magnesium sulfate.

\footnotetext{
1 Recibido: 7 de mayo, 2015. Aceptado: 2 de setiembre, 2015. Este trabajo forma parte de una investigación de la autora en el Instituto de Estudios para los Ecosistemas, Florencia, Italia.

2 Universidad de Costa Rica, Escuela de Biología, Centro de Investigación en Ciencias del Mar y Limnología, San Pedro, San José 2060, Costa Rica.ana.silva@ucr.ac.cr
} 


\section{INTRODUCCIÓN}

Las cianobacterias (procarioticas) y algas microscópicas (eucariotas) representan productores primarios importantes en la naturaleza y forman la base de la cadena alimenticia en ambientes acuáticos. Chlorella sorokiniana es una microalga verde unicelular, no flagelada, esférica, con 2-10 $\mu \mathrm{m}$ de diámetro, del phylum Chlorophyta (Schubert, 2003). Este género se ha estudiado ampliamente desde el punto de vista de producción de lípidos para biodiesel, biorremediación, suplemento alimenticio y en medicamentos (Becker, 2013; Borowitzka, 2013; Liu y $\mathrm{Hu}, 2013)$.

Con el propósito de obtener un buen crecimiento celular de las microalgas es indispensable el suministro de nutrimentos en cantidades adecuadas (Abalde et al., 1995; Grobbelar, 2013). Los medios nutritivos están compuestos básicamente de macronutrientes (nutrimentos esenciales para la formación estructural de las moléculas, p.ej. nitrógeno, carbono, oxígeno, fósforo, hidrógeno, magnesio, calcio, potasio y azufre), los elementos traza (micronutrientes que son adquiridos por las células en cantidades mínimas, necesarios para la activación de algunas enzimas, p.ej. hierro, manganeso, cobre, cobalto y molibdeno) y vitaminas (biotina, $\mathrm{B}_{12}$ y tiamina), preparados como soluciones patrones (Watanabe, 2005) y cuyas concentraciones varían principalmente del objetivo de estudio y la especie de microalga (Richmond, 1999). El carbono, nitrógeno y fósforo son considerados los nutrimentos limitantes para el crecimiento microalgal en aplicaciones biotecnológicas (Grobbelar, 2013).

Con el propósito de obtener productos de alto valor agregado como lípidos, proteínas, carbohidratos y pigmentos que pueden ser utilizados desde el punto de vista de la comercialización, es necesario producir biomasa a gran escala (Schenk et al., 2008; Liu y $\mathrm{Hu}, 2013)$. Este escalamiento a volúmenes mayores, implica una alta demanda de reactivos de óptimo grado analítico y un consumo considerable de hombre-tiempo que conlleva la preparación de los medios nutritivos, los cuales son costosos si se tienen que adquirir en grandes cantidades, lo que eleva los costos de producción de los cultivos de las microalgas (Molina et al., 2003; Simental y Sánchez, 2003; Borowizka, 2005). La preparación de los medios de cultivo a gran escala representa un $30-40 \%$ de los costos de operación, según Jad-Allah (2012), y entre 10-30\% según Borowitzka (1999). Por consiguiente, una alternativa es usar medios de enriquecimiento como los fertilizantes agrícolas; especialmente cuando se trabaja con volúmenes grandes de biomasa en ambientes externos, pues son económicos y contienen incorporados en su preparación macronutrientes, micronutrientes y vitaminas indispensables para el crecimiento de las microalgas (Richmond, 1999).

Estudios han demostrado la eficiencia de fertilizantes foliares como medios nutritivos en cultivos microalgales, cuyos resultados, en términos de productividad de la biomasa, son mayores o equivalentes con respecto a los medios nutritivos preparados a partir de reactivos químicos convencionales como el BG11 y F/2 (Nieves et al., 1996; Simental y Sánchez, 2003; Sánchez et al., 2008; Ortiz et al., 2011; Jad-Allah, 2012).

El objetivo del presente trabajo fue evaluar la productividad de la microalga verde $C$. sorokiniana UTEX 1230 con fertilizantes foliares usados en agricultura usados en la agricultura y la comparación con el medio nutritivo Kolwitz (K3).

\section{MATERIALES Y MÉTODOS}

\section{Diseño del experimento}

La investigación se realizó en el Instituto de Estudios para los Ecosistemas, Florencia, Italia, durante el año 2014. Se utilizó la microalga Chlorella sorokiniana UTEX 1230 disponible en la algoteca de este instituto.

Los experimentos se diseñaron como cultivos discontinuos. Para la preparación de cada experimento las células crecieron previamente en medio nutritivo Kolwitz (K3), se cosecharon en fase logarítmica, centrifugadas y lavadas tres veces con agua destilada para eliminar los nutrimentos contenidos en el medio original K3.

Se emplearon dos fertilizantes granulados utilizados comúnmente en la agricultura NPK 22 -10-7 (+2 MgO) y NPK 20-20-20. Ambos poseen en la composición química nitrógeno total $(\mathrm{N})$, óxido de fósforo $\left(\mathrm{P}_{2} \mathrm{O}_{5}\right)$ y óxido de potasio $\left(\mathrm{K}_{2} \mathrm{O}_{7}\right)$. Se usó el medio de cultivo convencional Kolkwitz (K3) como control (Concas et al., 2012). Los fertilizantes 
usados carecen de sulfato de magnesio y el fertilizante NPK 22-10-7 (+2 $\mathrm{MgO})$ posee en su composición óxido de magnesio (2\%). Los fertilizantes utilizados contienen microelementos como el boro, cobre, hierro, manganeso, zinc y molibdeno que son importantes para el crecimiento de la biomasa. El medio nutritivo K3 contiene en su fórmula química el compuesto de sulfato de magnesio. La caracterización y las concentraciones de cada especie química de los fertilizantes y el medio nutritivo K3 se describen en el Cuadro 1.

Se compararon cuatro tratamientos los cuales consistieron en la combinación de cada fertilizante con presencia y ausencia de sulfato de magnesio $\left(\mathrm{MgSO}_{4} 7 \mathrm{H}_{2} \mathrm{O}\right)$. Para preparar los tratamientos se agregó $1,00 \mathrm{~g} / \mathrm{l}$ de cada fertilizante en volúmenes de $400 \mathrm{ml}$ de agua destilada estéril. En los tratamientos con sulfato de magnesio se agregó $0,27 \mathrm{~g} / \mathrm{l}$ de este compuesto, equivalente a la concentración que se encuentra en el medio K3. Los tratamientos fueron los siguientes:

1) NPK $20-20-20(1,00 \mathrm{~g} / \mathrm{l})+0,27 \mathrm{~g} / 1 \mathrm{MgSO}_{4} \cdot 7 \mathrm{H}_{2} \mathrm{O}$

2) NPK 22-10-7 (+2MgO) $(1,00 \mathrm{~g} / \mathrm{l})+0,27 \mathrm{~g} / 1$ $\mathrm{MgSO}_{4} \cdot 7 \mathrm{H}_{2} \mathrm{O}$

3) NPK $22-10-7(+2 \mathrm{MgO})(1,00 \mathrm{~g} / \mathrm{l})$

Cuadro 1. Composición química de los fertilizantes agrícolas NPK 22-10-7 (+2 MgO), NPK 20-20-20 y medio nutritivo Kolwitz (K3). Italia. 2014.

Table 1. Chemical composition of agricultural fertilizers NPK 22-10-7 (+2 MgO), NPK 20-20-20 and nutrient medium Kolwitz (K3). Italy. 2014.

\begin{tabular}{|c|c|c|}
\hline $\begin{array}{c}\text { NPK 22-10-7 (+2MgO) } \\
\text { Microelementos y magnesio }\end{array}$ & $\begin{array}{l}\text { NPK 20-20-20 } \\
\text { Microelementos }\end{array}$ & $\begin{array}{c}\text { Kolwitz (K3) } \\
\text { Microelementos y sulfato de magnesio }\end{array}$ \\
\hline $\begin{array}{c}\text { Nitrógeno total: } 22 \% \text { ( } 8,4 \% \text { nítrico; } \\
3,6 \% \text { urea, } 10 \% \text { amonio) }\end{array}$ & $\begin{array}{c}\text { Nitrógeno total: } 20 \% \text { (6\% nítrico, } \\
8,8 \% \text { urea, } 5,2 \% \text { amonio) }\end{array}$ & Nitrógeno total: $9 \%$ \\
\hline $\begin{array}{c}\mathrm{P}_{2} \mathrm{O}_{5} 100,00 \mathrm{~g} / \mathrm{l} \\
(\mathrm{P}=4,3 \%)\end{array}$ & $\begin{array}{c}\mathrm{P}_{2} \mathrm{O}_{5} 200,00 \mathrm{~g} / \mathrm{l} \\
(\mathrm{P}=8,7 \%)\end{array}$ & $\begin{array}{c}\mathrm{K}_{2} \mathrm{HPO}_{4} \quad 0,420 \mathrm{~g} / \mathrm{l} \\
(\mathrm{P}=3 \%) \\
(\mathrm{K}=7 \%)\end{array}$ \\
\hline $\begin{array}{c}\mathrm{MgO} 20,00 \mathrm{~g} / \mathrm{l} \\
(\mathrm{Mg}=1,2 \%)\end{array}$ & $1,00 \mathrm{~g} / \mathrm{l}$ & $\begin{array}{c}\mathrm{Mg} \mathrm{SO}_{4} 7 \mathrm{H}_{2} \mathrm{O} 0,270 \mathrm{~g} / \mathrm{l} \\
(\mathrm{Mg}=1,10 \%) \\
(\mathrm{S}=1,43 \%)\end{array}$ \\
\hline $\begin{array}{c}\mathrm{K}_{2} \mathrm{O} 70,00 \mathrm{~g} / \mathrm{l} \\
(\mathrm{K}=5,7 \%)\end{array}$ & $\begin{array}{c}\mathrm{K}_{2} \mathrm{O} 200,00 \mathrm{~g} / 1 \\
(\mathrm{~K}=16,5 \%)\end{array}$ & $\begin{array}{c}\mathrm{KNO}_{3} 1,710 \mathrm{~g} / \mathrm{l} \\
(\mathrm{K}=26 \%)\end{array}$ \\
\hline \multicolumn{3}{|c|}{ Microelementos } \\
\hline B $0,10 \mathrm{~g} / 1$ & $\begin{array}{l}\text { B } 0,50 \mathrm{~g} / 1 \\
\mathrm{Cu} 0,10 \mathrm{~g} / 1\end{array}$ & $\begin{array}{cc}\mathrm{H}_{3} \mathrm{BO}_{3} & 2,860 \mathrm{~g} / 1 \\
\mathrm{CuSO}_{4} 5 \mathrm{H}_{2} \mathrm{O} & 0,080 \mathrm{~g} / 1\end{array}$ \\
\hline Cu-EDTA $0,10 \mathrm{~g} / \mathrm{l}$ & Cu-EDTA $0,10 \mathrm{~g} / 1$ & \\
\hline Fe-EDTA $1,60 \mathrm{~g} / 1$ & $\begin{array}{c}\mathrm{Fe} 2,00 \mathrm{~g} / \mathrm{l} \\
\mathrm{Mn} \quad 1,00 \mathrm{~g} / \mathrm{l}\end{array}$ & $\begin{array}{cc}\mathrm{CoCl}_{2} .5 \mathrm{H}_{2} \mathrm{O} & 0,035 \mathrm{~g} / \mathrm{l} \\
\mathrm{Fe}_{2}\left(\mathrm{SO}_{4}\right)_{3} 7 \mathrm{H}_{2} \mathrm{O} & 24,900 \mathrm{~g} / \mathrm{l} \\
\mathrm{MnSO}_{4} \cdot 4 \mathrm{H}_{2} \mathrm{O} & 1,810 \mathrm{~g} / \mathrm{l}\end{array}$ \\
\hline Mn EDTA $0,60 \mathrm{~g} / 1$ & $\begin{array}{l}\text { Mn-EDTA } 1,00 \mathrm{~g} / 1 \\
\text { Zn } 0,10 \mathrm{~g} / 1\end{array}$ & $\mathrm{ZnSO}_{4} \cdot 7 \mathrm{H}_{2} \mathrm{O} \quad 0,222 \mathrm{~g} / 1$ \\
\hline Zn-EDTA $0,10 \mathrm{~g} / 1$ & Zn-EDTA $0,10 \mathrm{~g} / 1$ & EDTA-Na $\quad 29,750 \mathrm{~g} / 1$ \\
\hline $\begin{array}{c}\text { Mo-EDTA } 0,06 \mathrm{~g} / 1 \\
\mathrm{pH}=2,01-3,21\end{array}$ & $\begin{array}{c}\text { Mo } 0,05 \mathrm{~g} / 1 \\
\mathrm{pH}=7,02-7,50\end{array}$ & $\begin{array}{c}\mathrm{Na}_{2} \mathrm{MoO}_{4} \cdot 2 \mathrm{H}_{2} \mathrm{O} \quad 0,230 \mathrm{~g} / 1 \\
\mathrm{pH}=7,20-7,50\end{array}$ \\
\hline
\end{tabular}


4) NPK $20-20-20(1,00 \mathrm{~g} / \mathrm{l})$

5) K3 (control)

\section{Métodos analíticos}

Cada experimento se realizó cinco veces con tres tubos tipo biorreactor en volúmenes de 400 $\mathrm{ml}$ cada uno. Las mediciones se iniciaron con una concentración inicial de clorofila $a$ de $4,00 \mathrm{mg} / \mathrm{l}$ en el inoculo, expuestos a una temperatura de $28{ }^{\circ} \mathrm{C}$ con una intensidad lumínica artificial de $150 \mu$ mol fotones $\mathrm{m}^{2} / \mathrm{s}$ provista con tubos blancos fluorescentes. A cada cultivo se le incorporó $\mathrm{CO}_{2} /$ aire en una relación 2:98 $v / v$ y un flujo constante de $5 \mathrm{l} / \mathrm{min}$. El pH se midió cada veinticuatro horas y se mantuvo en un valor de 7,0 . En caso de una disminución del $\mathrm{pH}$, este se ajustó con $\mathrm{NaOH} 1 \mathrm{M}$ estéril. Para evitar variaciones, todos los experimentos se realizaron en las mismas condiciones iniciales de concentración de clorofila, temperatura e intensidad lumínica.

\section{Medición del crecimiento y productividad}

El crecimiento en términos de peso seco $(\mathrm{g} / \mathrm{l})$ se cuantificó a partir de volúmenes de $10 \mathrm{ml}$ (en duplicado) de cada experimento, con filtros prepesados $(\mathrm{GF} / \mathrm{F})$. Los filtros con las muestras se secaron a una temperatura de $105{ }^{\circ} \mathrm{C}$ durante tres horas y se pesaron en una balanza de $0,01 \mathrm{mg}$ de precisión. La productividad $(\mathrm{mg} / \mathrm{l} / \mathrm{h})$ se calculó según la siguiente ecuación, $x_{1}-x_{0 /} \Delta t$, donde $x_{1}$ y $x_{0}(\mathrm{mg} / \mathrm{l} / \mathrm{h})$ corresponden a la diferencia entre el peso seco al final e inicio del experimento y $\Delta t$ es el número total de horas.

Pigmentos: clorofila total (mg/l)

La clorofila total (mg/l) se extrajo con acetona $90 \%$ (Lichenthaler, 1987) y se midió con un espectrofotómetro.

\section{Fluorescencia de la clorofila $\left(\mathrm{F}_{\mathrm{v}} / \mathrm{F}_{\mathrm{m}}\right)$}

La fluorescencia de la clorofila $\left(\mathrm{F}_{\mathrm{v}} / \mathrm{F}_{\mathrm{m}}=\right.$ relación entre la fluorescencia variable $F_{v}$ con respecto a la fluorescencia máxima $\mathrm{F}_{\mathrm{m}}$, es una herramienta confiable para estudiar el estado fisiológico de las plantas y microalgas (Krause y Weis, 1984; Torzillo et al., 2012; Masojídek et al., 2013; Torzillo y Vonshak, 2013). Para la medición del $\mathrm{F}_{\mathrm{v}} / \mathrm{F}_{\mathrm{m}}$ cada muestra se adaptó en la oscuridad durante diez minutos; luego se colocó un volumen de $0,50 \mathrm{ml}$ en una cámara de $10 \mathrm{~mm}$ de longitud y se midió con un fluorómetro tipo PAM (Silva et al., 2013).

\section{Composición de la biomasa}

Las proteínas se determinaron con el método de Lowry y Rosenbrough (1951), previa extracción con hidróxido de sodio 0,1 N. Los carbohidratos se cuantificaron con el método según Dubois et al. (1956). Los estándares usados en las curvas de calibración correspondieron a albúmina de bovino para la determinación de proteínas y glucosa anhidra para carbohidratos.

\section{Análisis estadístico}

Los análisis estadísticos se realizaron con el paquete estadístico Stat Graphics Plus, versión 5.1 para Windows. Las diferencias fueron examinadas por medio de un análisis de variancia (ANOVA) de una vía, con pruebas post-hoc de Tuckey.

\section{RESULTADOS Y DISCUSIÓN}

Los resultados indicaron que el crecimiento, la productividad, pigmentos, fluorescencia y composición bioquímica de $C$. sorokiniana estuvieron influenciadas por la presencia de sulfato de magnesio, donde los mayores valores se observaron en los tratamientos que presentaron este compuesto. Las microalgas presentaron un patrón de crecimiento que involucró las fases de adaptación, logarítmica, estacionaria y declinación (Arredondo y Voltolina, 2007; Lee et al., 2013) (Figura 1).

El mayor crecimiento (fase exponencial), se midió entre el cuarto y sexto día $(3,25 \mathrm{~g} / \mathrm{l})$ en el cultivo con el tratamiento NPK 20-20-20 (1,00 g/l $)+0,27 \mathrm{~g} / \mathrm{l}$ $\mathrm{MgSO}_{4} \cdot 7 \mathrm{H}_{2} \mathrm{O}$ y entre el sexto y octavo día $(2,24 \mathrm{~g} / \mathrm{l})$ en el cultivo con NPK 22-10-7 (+2 MgO) $(1,00 \mathrm{~g} / \mathrm{l})+$ $0,27 \mathrm{~g} / \mathrm{l} \mathrm{MgSO}_{4} \cdot 7 \mathrm{H}_{2} \mathrm{O}$. El cultivo con el control (K3) presentó el crecimiento más alto $(3,65 \mathrm{~g} / \mathrm{l})$, con una tendencia de crecimiento similar al primer tratamiento. Los tratamientos ausentes de sulfato de magnesio mostraron una fase logarítmica de uno-dos días, con un crecimiento menor de $1,00 \mathrm{~g} / \mathrm{l}$, y declinando entre el tercer y cuarto día, manifestando un color amarillento. 


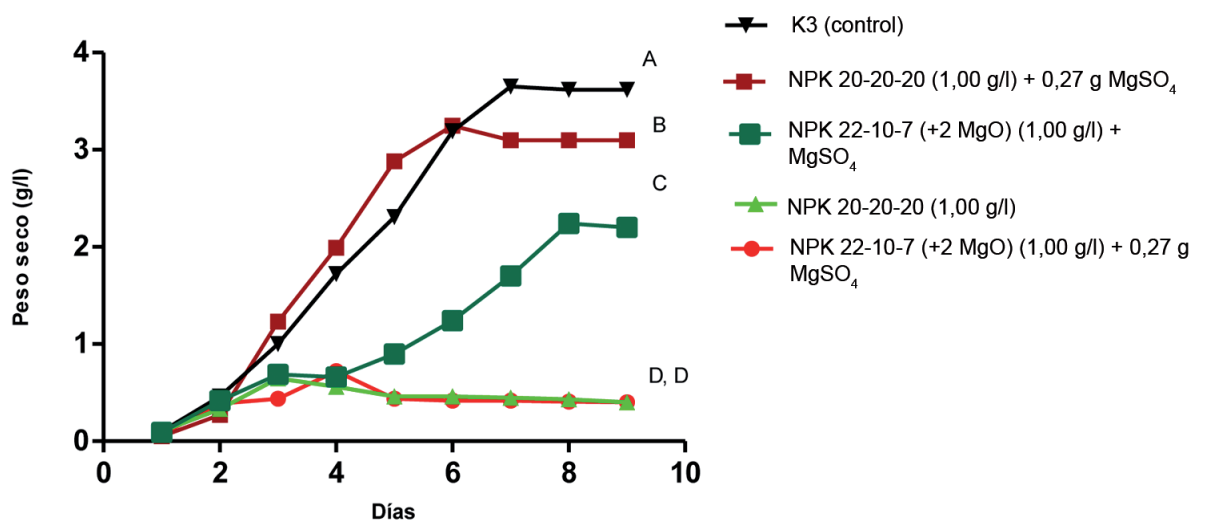

Figura 1. Curvas de crecimiento (g/l) del cultivo de Chlorella sorokiniana UTEX 1230 en cuatro tratamientos con dos fertilizantes agrícolas. Italia. 2014.

Letras diferentes en cada curva indican diferencia significativa a $\mathrm{P} \leq 0,05$ una vía ANOVA.

Figure 1. Growth curves (g/l) for Chlorella sorokiniana UTEX 1230 culture at four treatments with two agricultural fertilizers. Italy. 2014.

Curves denoted by different letters differ significantly at $\mathrm{P}<0.05$ one-way ANOVA.

En términos de productividad, los cultivos con sulfato de magnesio presentaron los mayores valores con respecto a los cultivos ausentes de este compuesto $(\mathrm{P} \leq 0,01)$, donde el cultivo con el tratamiento NPK 20-20-20 (1,00 g/l) + 0,27 g/l $\mathrm{MgSO}_{4} \cdot 7 \mathrm{H}_{2} \mathrm{O}$ fue el que mostró la productividad más alta (Figura 2).
El cultivo con la combinación del fertilizante NPK 20-20-20 y sulfato de magnesio, resultó el tratamiento con la mayor concentración de clorofila total $(\mathrm{mg} / \mathrm{l})$, aunque significativamente menor que el control K3 $(\mathrm{P} \leq 0,01)$. Los cultivos que presentaron menor concentración de pigmentos correspondieron a

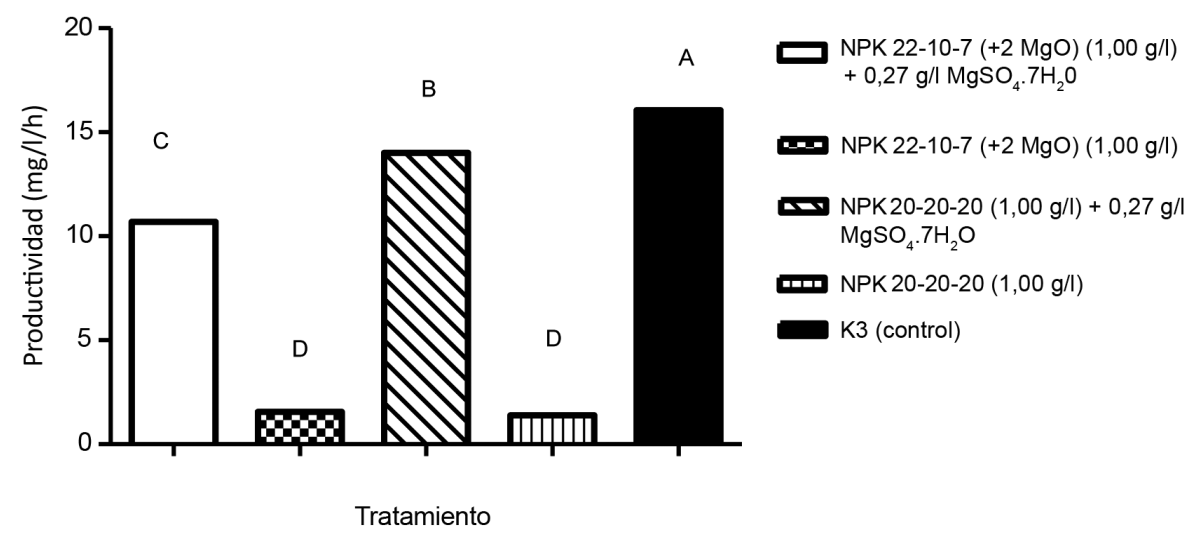

Figura 2. Productividad $(\mathrm{mg} / \mathrm{l} / \mathrm{h})$ del cultivo de Chlorella sorokiniana UTEX 1230 en cuatro tratamientos con dos fertilizantes agrícolas. Italia. 2014.

Letras diferentes en cada curva indican diferencia significativa a $\mathrm{P} \leq 0,05$ una vía ANOVA.

Figure 2. Productivity (mg/l/h) of Chlorella sorokiniana UTEX 1230 culture at four treatments with two agricultural fertilizers. Italy. 2014.

The bars denoted by different letters differ significantly at $\mathrm{P}<0.05$ one-way ANOVA. 
los tratamientos que no contienen sulfato de magnesio. Relacionado con esta observación, Mellis et al. (2000), reportaron una disminución en la actividad fotosintética en cultivos de microalgas deprovistos de azufre; por consiguiente, para evitar un severo daño oxidativo en las células de las microalgas en ausencia de este elemento (Torzillo et al., 1996), y con ello una reducción en el metabolismo general de las células y composición bioquímica de la biomasa, es importante la adición de sulfato en la fórmula química del medio nutritivo. Los resultados mostraron que los mayores porcentajes de carbohidratos y proteínas correspondieron a los tratamientos con sulfato de magnesio, comparables con la biomasa creciendo en el medio nutritivo K3; mientras que los valores menores se evidenciaron en los cultivos ausentes de este compuesto, situación que se reflejó en el color amarillento que presentó la biomasa, indicando una oxidación del cultivo (Cuadro 2).

Con el propósito de monitorear la actividad de los cultivos en los diferentes tratamientos, se realizaron mediciones de fluorescencia de la clorofila (Fv/Fm). Los cambios más importantes se observaron en una disminución de esta variable y en la productividad en los cultivos desprovistos de sulfato de magnesio $(\mathrm{P} \leq 0,01)$ con respecto a los tratamientos provistos de azufre donde alcanzaron valores de $\mathrm{F}_{\mathrm{v}} / \mathrm{F}_{\mathrm{m}}$ cercanos o iguales a 0,7 y semejantes con el cultivo control (K3) $(\mathrm{P}=0,01)$ (Figura 3). Los bajos valores en la relación $\mathrm{F}_{\mathrm{v}} /$ $\mathrm{F}_{\mathrm{m}}$ en los tratamientos sin sulfato $(0,43$ en NPK 20-20$20(1,00 \mathrm{~g} / \mathrm{l})$ y 0,55 en NPK $22-10-7(+2 \mathrm{MgO})(1,00$ $\mathrm{g} / \mathrm{l})$ indicaron un "stress" fotohinbitorio en los cultivos, que se reflejó en el color amarillento de la biomasa en los últimos días del experimento (Figura 3).

El magnesio $\left(\mathrm{Mg}^{2+}\right)$ se encuentra incorporado en la fórmula química del fertilizante NPK 22-10-7 y se encuentra asociado a la estructura de la clorofila como ión magnesio $\left(\mathrm{Mg}^{2+}\right)$, aspecto fundamental en la función de esta molécula. La importancia de este ión se evidencia en la mayor concentración de clorofila y fluorescencia en el tratamiento con el fertilizante NPK $22-10-7(+2 \mathrm{MgO})\left(\mathrm{F}_{\mathrm{v}} / \mathrm{F}_{\mathrm{m}} 0,55\right)$ en ausencia de sulfato, con respecto al tratamiento con el fertilizante NKP 2020-20 (1,00 g/l) ( $\left.\mathrm{F}_{\mathrm{v}} / \mathrm{F}_{\mathrm{m}} 0,43\right)$ carente de este compuesto.

Además del sulfuro en los fertilizantes utilizados, es importante considerar la presencia de diferentes iones de nitrógeno, especialmente amonio $\left(\mathrm{NH}_{4}^{+}\right)$y urea $\left(\mathrm{CH}_{4} \mathrm{~N}_{2} \mathrm{O}\right)$. La composición y la concentración del nitrógeno, ya sea en forma de amonio, urea, nitrito y nitrato, afectan el valor del $\mathrm{pH}$ del medio nutritivo (Silva y Torzillo, 2012), el crecimiento y la productividad de la biomasa, contenido de proteínas, carbohidratos, clorofila y fluorescencia de las células (Becker, 1995; Grobbelaar, 2013). Los fertilizantes usados contienen en su composición altas concentraciones de nutrimentos inorgánicos, especialmente nitrógeno, el cual se encuentra en diversas formas como óxido nítrico, urea y nitrógeno de amonio $\left(\mathrm{N}_{-} \mathrm{NH}_{4}^{+}\right)$en diferentes proporciones (Cuadro 1). Tam y Wong (1990) han observado la asimilación de amonio y nitrito en la especie Chlorella pyrenoidosa removiendo de esta manera nutrimentos del medio. Estudios realizados con la microalga Dunaliella sp. reportan buenas concentraciones de

Cuadro 2. Valores promedio y desviación estándar de las proteínas (\%) y carbohidratos (\%) de Chlorella sorokiniana UTEX 1230 en dos medios de fertilizantes evaluados. Italia. 2014.

Table 2. Mean and standard deviations of proteins (\%) and carbohydrates (\%) of Chlorella sorokiniana UTEX 1230 in two evaluated fertilizer media. Italy. 2014.

\begin{tabular}{lcc}
\hline Tratamiento & Proteínas (\%) & Carbohidratos (\%) \\
\hline Kolwitz (K3) Control & $52,08 \pm 0,07 * * *$ & $38,04 \pm 0,05 * * *$ \\
$20-20-20(1,00 \mathrm{~g} / \mathrm{l})+0,27 \mathrm{~g} / 1 \mathrm{MgSO}_{4} \cdot 7 \mathrm{H}_{2} \mathrm{O}$ & $50,10 \pm 0,10 * * *$ & $37,32 \pm 0,27 * * *$ \\
$22-10-7(+2 \mathrm{MgO}) 1,00 \mathrm{~g} / \mathrm{l}+0,27 \mathrm{~g} / 1 \mathrm{MgSO}_{4} \cdot 7 \mathrm{H}_{2} \mathrm{O}$ & $48,40 \pm 0,36 * * *$ & $35,12 \pm 0,10 * * *$ \\
$20-20-20(1,00 \mathrm{~g} / \mathrm{l})$ & $4,10 \pm 0,10^{* *}$ & $2,05 \pm 0,00 \mathrm{n} . \mathrm{s}$ \\
$22-10-7(+2 \mathrm{MgO}) 1,00 \mathrm{~g} / 1$ & $3,24 \pm 0,25 * *$ & $1,98 \pm 0,00 \mathrm{n} . \mathrm{s}$ \\
\hline
\end{tabular}

El nivel de significancia de las pruebas corresponde al $\mathrm{p}<0,05$, n.s $=$ no significativo $/$ The significance level of the test was $\mathrm{p}<0.05$ one-way ANOVA, n.s. = not significant. 


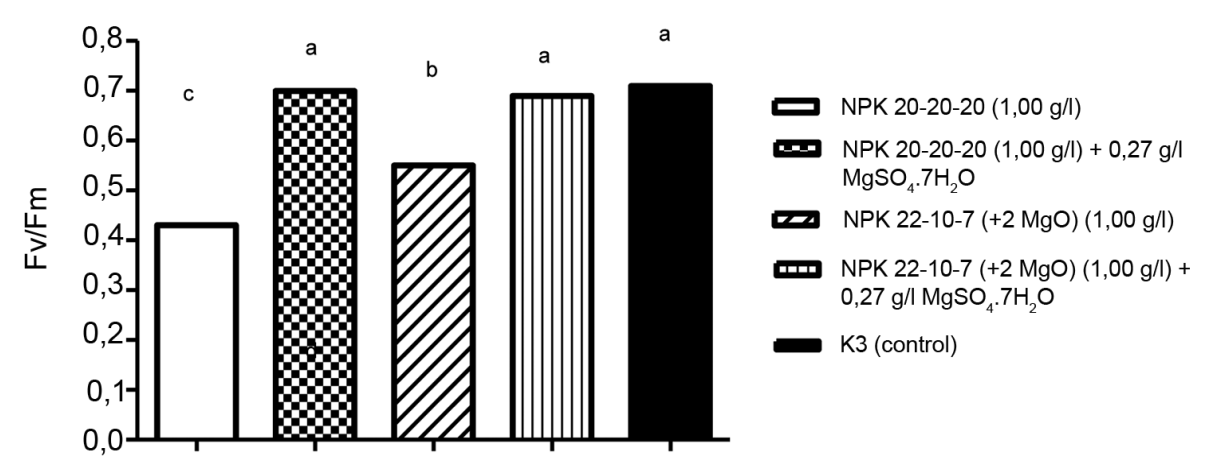

Tratamiento

Figura 3. Fluorescencia de la clorofila $a(\mathrm{Fv} / \mathrm{Fm})$ del cultivo de Chlorella sorokiniana UTEX 1230 en cuatro tratamientos con dos fertilizantes agrícolas. Italia. 2014.

Letras diferentes en cada curva indican diferencia significativa a $\mathrm{P} \leq 0,05$ una vía ANOVA.

Figure 3. Chlorophyll a fluorescence $(\mathrm{Fv} / \mathrm{Fm})$ of Chlorella sorokiniana UTEX 1230 culture at four different treatments with two agricultural fertilizers. Italy. 2014.

Bars denoted by different letters differ significantly at $\mathrm{P}<0.05$ one-way ANOVA.

células en medios nutritivos ricos de nutrimentos (Becerra-Dórame et al., 2010) y una alta densidad de Nannochloropsis gaditana en el medio F/2 enriquecido con nitrato (Converti et al., 2009).

El fertilizante NPK 22-10-7 contiene en su composición un $10 \%$ de nitrógeno-amonio, equivalente a una concentración de $100 \mathrm{mg} / \mathrm{l}$, mientras que el fertilizante NPK 20-20-20 posee un 5,20\% de este ión $(50 \mathrm{mg} / \mathrm{l})$, y aunque es la forma preferida de nitrógeno para la asimilación por parte de las microalgas (Abalde et al., 1995; Becker, 1995), cuando se presenta en altas concentraciones, el $\mathrm{pH}$ del medio disminuye significativamente (Silva y Torzillo, 2012; Grobbelaar, 2013). En Chlorella vulgaris, concentraciones superiores a $80 \mathrm{mg} / \mathrm{l}$ de amonio, disminuyeron el crecimiento y productividad celular (Tam y Wong, 1996). En el presente trabajo la disminución del pH en el cultivo con el fertilizante NPK 22-10-7 ( $\mathrm{pH}=2,02-$ 3,21 ), debido al alto contenido de $\mathrm{N}_{-} \mathrm{NH}_{4}^{+}$, incidió en una disminución en el crecimiento celular. Las microalgas presentan un $\mathrm{pH}$ óptimo entre 7,00-8,00 y una reducción a niveles menores de 6,00 puede ser letal para los organismos (Abalde et al., 1995; Silva y Torzillo, 2012).

La presencia de urea $\left(\mathrm{CH}_{4} \mathrm{~N}_{2} \mathrm{O}\right)$ en el medio también es importante para el crecimiento y productividad del cultivo; es una fuente de nitrógeno apta para la producción de microalgas, pues estas requieren menos energía para su asimilación en comparación con el nitrito o nitrato; además, es más económico que estos dos compuestos con sus respectivas ventajas para los cultivos a gran escala (Becker, 1995). El fertilizante NPK 20-20-20 contiene una cantidad doblemente superior de este elemento $(8,80 \%)$ que el NPK $22-$ 10-7 (3,60\%) (Cuadro 1), situación que beneficia un aumento en la tasa de crecimiento de la biomasa en el cultivo con el primer fertilizante, favorecido también por el contenido de carbono en la urea que promueve los procesos fotosintéticos de las células de las microalgas. El efecto positivo de la urea sobre otras fuentes de nitrógeno en el crecimiento de microalgas como Nannochloropsis sp. y diatomeas marinas ha sido confirmado por Rocha et al. (2003), Piña et al. (2007) y Jad-Allah (2012).

Una consideración para usar un medio nutritivo diferente al K3, es asegurar la posibilidad de obtener buen crecimiento y productividad celular en un medio nutritivo más económico que el convencional. El costo de los reactivos químicos para cada uno de los tratamientos utilizados en el experimento e hipotizando una producción máxima de $60000 \mathrm{~kg} / \mathrm{ha} /$ día (Tredici, 2010) en cultivos masivos de biomasa con el medio nutritivo K3, se presentan en el Cuadro 3.

K3 (Kolkwitz): para el cálculo con este medio nutritivo se consideró las principales soluciones "madre": nitrato de potasio $\left(\mathrm{KNO}_{3}\right)$, potasio hidrógeno 
Cuadro 3. Costos de los tratamientos $\mathrm{K} 3,20-20-20(1,00 \mathrm{~g} / \mathrm{l})+0,27 \mathrm{~g} / 1 \mathrm{MgSO} \cdot 7 \mathrm{H}_{2} \mathrm{O}$ y $22-10-7(+2 \mathrm{MgO}) 1,00 \mathrm{~g} / \mathrm{l}+0,27 \mathrm{~g} / \mathrm{l}$ $\mathrm{MgSO}_{4} \cdot 7 \mathrm{H}_{2} \mathrm{O}$ para una producción hipotética de $60000 \mathrm{~kg} / \mathrm{ha}$ /día de cultivo masivo de microalgas. Italia. 2014.

Table 3. Costs of treatments, 20-20-20 (1,00 g/l $)+0,27 \mathrm{~g} / 1 \mathrm{MgSO}_{4} \cdot 7 \mathrm{H}_{2} \mathrm{O}$ and 22-10-7 (+2MgO) $1,00 \mathrm{~g} / 1+0,27 \mathrm{~g} / 1 \mathrm{MgSO}$. $7 \mathrm{H}_{2} \mathrm{O}$ for a hypothetical output of $60000 \mathrm{~kg} / \mathrm{ha} / \mathrm{day}$ of mass cultivation of microalgae in bioreactors. Italy. 2014.

\begin{tabular}{|c|c|c|c|c|}
\hline Tratamiento & $\begin{array}{c}\text { Productividad } \\
(\%)\end{array}$ & $\begin{array}{c}\text { Producción } \\
\text { (kg/ha/día) }\end{array}$ & $\begin{array}{c}\text { Costo del } \\
\text { tratamiento para } \\
\text { producir } 1 \mathrm{~kg} \mathrm{de} \\
\text { biomasa }(\$)\end{array}$ & $\begin{array}{c}\text { Superficie de terreno } \\
\text { necesario para } \\
\text { producir } 60000 \mathrm{~kg} / \\
\text { ha/día }\left(\mathrm{m}^{2}\right)\end{array}$ \\
\hline K3 & 100 & 60000 & 7,28 & 10,000 \\
\hline $20-20-20(1,00 \mathrm{~g} / \mathrm{l})+0,27 \mathrm{~g} / \mathrm{lMgSO}_{4} \cdot 7 \mathrm{H}_{2} \mathrm{O}$ & 90 & 54000 & 4,01 & 11,400 \\
\hline $22-10-7(+2 \mathrm{MgO}) 1,00 \mathrm{~g} / 1+0,27 \mathrm{~g} / 1 \mathrm{MgSO}_{4} \cdot 7 \mathrm{H}_{2} \mathrm{O}$ & 67 & 40200 & 1,16 & 15,000 \\
\hline
\end{tabular}

de fosfato $\left(\mathrm{K}_{2} \mathrm{HPO}_{4}\right)$ y sulfato de magnesio $\left(\mathrm{MgSO}_{4}\right.$ $\left.7 \mathrm{H}_{2} \mathrm{O}\right)$. No se cuantificaron los reactivos que conforman los microelementos.

Fertilizantes: se incluye únicamente en el análisis de costos los tratamientos que se les agregó sulfato de magnesio.

Los cálculos incluyen el costo de los reactivos, no considera variables como mano de obra, tiempo de preparación, consumo del dióxido de carbono, equipo de laboratorio, tamaño y materiales del biorreactor.

Los resultados en el laboratorio demostraron que el tratamiento NPK 20-20-20 (1,00 g/l) + 0,27 $\mathrm{g} / \mathrm{l} \mathrm{MgSO}_{4} \cdot 7 \mathrm{H}_{2} \mathrm{O}$ presentó un $90 \%$ de productividad y el NPK 22-10-7 (+2 MgO) 1,00 g/l + 0,27 g/l $\mathrm{MgSO}_{4} \cdot 7 \mathrm{H}_{2} \mathrm{O}$ un $67 \%$ con respecto al K3 (Figura 2). Extrapolando estas productividades a cultivos masivos en biorreactores, se necesitaría una producción de $54000 \mathrm{~kg} / \mathrm{ha} /$ día en el primer tratamiento y 40 $200 \mathrm{~kg} / \mathrm{ha} /$ día en el segundo tratamiento. En el Cuadro 3 se observa que para producir un kilo de biomasa el costo es mayor en el cultivo con medio nutritivo convencional, mientras que el más económico es el tratamiento con el fertilizante NPK 22-10-7, reflejando este último, un ahorro de un $84 \%$ con respecto a un $45 \%$ con el fertilizante NPK 2020-20, si se compara con el precio del medio K3. Si se considera el significativo ahorro en términos de dinero que representa usar el fertilizante NPK 22-107 , no obstante la disminución de la productividad del cultivo que presentó este tratamiento, el bajo costo del fertilizante podría ser considerado una ventaja para usarlo en cultivos masivos en condiciones externas. Es importante considerar que aún el fertilizante NPK 20-
20-20 fue más económico que el cultivo con el medio convencional K3 y presentó una productividad alta, comparable con este último medio, resultado que lo hace atractivo para usarlo en cultivos masivos.

La incidencia del costo del tratamiento sobre el costo total de la producción microalgal en el presente estudio (Acién et al. 2012), corresponde para el tratamiento con $\mathrm{K} 3$ un 48,50\%, para el NKP 20-2020 un $26,73 \%$ y para el fertilizante NKP 22-10-7 un valor de $7,70 \%$. Este último dato coincide con los valores de incidencia reportados por Norsker et al. (2011), los cuales se encuentran en un ámbito entre 4,6-10\%. Adicionalmente, se observa en el Cuadro 3 el dato calculado para la cantidad de terreno $\left(\mathrm{m}^{2}\right)$ necesario para producir $60000 \mathrm{~kg} / \mathrm{ha} /$ día con cada tratamiento. Es evidente que con el NPK 22-10-7 (+2 $\mathrm{MgO}) 1,00 \mathrm{~g} / \mathrm{l}+0,27 \mathrm{~g} / \mathrm{MgSO}_{4} .7 \mathrm{H}_{2} \mathrm{O}$ se necesitaría invertir en una cantidad mayor de terreno, aspecto a considerar cuando se realiza un análisis de costos en una producción masiva de microalgas.

La preparación de los medios nutritivos basados en fertilizantes conllevan a una reducción de tiempo comparado con $\mathrm{K} 3$, ya que para preparar este último, se deben pesar once reactivos diferentes, mientras que con fertilizantes se realiza solo una pesada, pues todos los reactivos están incorporados en la fórmula química del fertilizante.

Aunque el uso de fertilizantes se propone como una alternativa con respecto a los medios nutritivos convencionales para la producción de la biomasa en biorreactores a gran escala, en la contabilidad total, se debe cuantificar no solamente el costo de los reactivos, sino también la productividad de la biomasa, 
aspecto importante para la rentabilidad del cultivo, especialmente si se cultiva en volúmenes grandes para propósitos comerciales. Otros aspectos a considerar son el costo del terreno, mano de obra, electricidad, agua, dióxido de carbono, material y tamaño del biorreactor, costos de mantenimiento, objetivo de la producción (uso comestible, medicinal, fertilizante, biodiesel), aspectos que pueden variar el costo.

La reducción de los costos de producción de los medios nutritivos basados en fertilizantes agrícolas no se reflejó en un detrimento en la productividad ni en los componentes bioquímicos de la biomasa microalgal. Una buena producción de biomasa se obsevó en el alga T. suecica creciendo en fertilizantes foliares (Fábregas et al., 1987). Un buen crecimiento celular y altos valores de proteínas, carbohidratos y lípidos comparables al medio nutritivo f2 se reportó en cultivos de Chaetoceros muelleri, Thalassiosira weissflogii, Isochrysis sp. y Tetraselmis suecica (Piña et al., 2007). Por otra parte, un buen crecimiento y una óptima composición bioquímica se demostró en diatomeas bentónicas marinas y Rhodomonas sp. creciendo al utilizar fertilizantes comerciales (Simental et al., 2001; Simental y Sánchez, 2003; Valenzuela et al., 2005).

Los resultados indicaron buenos rendimientos en los cultivos de $C$. sorokiniana con fertilizantes agrícolas comerciales en condiciones de laboratorio. Se evidencia que para obtener un buen crecimiento y productividad de la biomasa microalgal, una adecuada concentración de clorofila, proteínas y carbohidratos en esta microalga, se debe asegurar que el fertilizante usado contenga una adecuada combinación de urea, amonio y elementos como potasio, fósforo, magnesio, sulfuro, micronutrientes, vitaminas y un aporte parcial de $\mathrm{CO}_{2}$ que ayudaría a estabilizar el $\mathrm{pH}$.

\section{LITERATURA CITADA}

Abalde, J., A. Cid, P. Fidalgo, E. Torres, y C. Herrero. 1995. Microalgas: cultivos y aplicaciones. La Coruña: Universidade Da Coruña, ESP.

Acién, F.G., J. M. Fernández, J.J. Magán, and E. Molina. 2012. Production cost of a real microalgae production plant and strategies to reduce it. Biotechnol. Adv. 30: 1344-1353.

Arredondo, B.O., y D. Voltolina. 2007. Concentración, recuento celular y tasa de crecimiento. En: B.
Arredondo, y D. Voltolina, editores, Métodos y herramientas analíticas en la evaluación de la biomasa microalgal. Centro de Investigaciones Biológicas del Noroeste, S.C. La Paz, CA, USA. p. 17-26.

Becerra-Dórame, M., J. A. López, F. Enríquez, N. Huerta, D. Voltolina, I. Osuna, and G. Izaguirre. 2010. The effect of initial cell and nutrient concentrations on the growth and biomass production of outdoor cultures of Dunaliella sp. Ann. Bot. Fennici. 47:109-112.

Becker, E. 1995. Microalgae: Biotechnology and microbiology. Cambridge University Press, USA.

Becker, W. 2013. Microalgae for human and animal nutrition. In: A. Rhichmond, and Q. Hu, editors, Handbook of microalgal culture: applied phycology and biotechnology. John Wiley y Sons, Ltd. GBR. p. 461-503.

Borowizka, M.A. 1999. Economic evaluation of microalgal processes and products. In: Z. Cohen, editor, Chemicals from microalgae. T.J. International Ltd, Padstow, GBR. p. 387-409.

Borowitzka, M.A. 2005. Culturing microalgae in outdoor ponds. En: R.A. Andersen, editor, Algal culturing techniques. Elsevier Academic Press, CA, USA. p. 205-218.

Borowitzka, M. 2013. High-value products from microalgaetheir development and commercialization. J. Appl. Phycol. 25:743-756.

Concas, A., G. Lutzu, M. Pisu, and G. Cao. 2012. Experimental analysis and novel modeling of semibatch photobioreactorsoperated with Chlorella vulgaris and fed with $100 \%(\mathrm{v} / \mathrm{v}) \mathrm{CO}_{2}$. Chem. Eng. J. 213:203-213.

Converti, A., A. Casazza, E. Ortiz, P. Perego, and Del Borghi. 2009. Effect of temperature and nitrogen concentration on the growth and lipid content of Nannochloropsis oculata and Chlorella vulgaris for biodiesel production. Chem. Eng. Process. 48:1146-1151.

Dubois, M., K. Guilles, J. Rebers, and F. Smith. 1956. Colorimetric method for determination of sugar and related substances. Anal. Chem. 28:350-356.

Fábregas, J., L. Toribio, J. Abalde, B. Cabezas, and C. Herrero. 1987. Approach to biomass production of the marine microalgae Tetraselmis suecica (Kylin) using common garden fertilizer and soil extract as cheap nutrient supply in batch cultures. Aquacult. Eng. 6:141-150.

Grobbelar, J. 2013. Inorganic algal nutrition. In: A. Rhichmond, and Q. Hu, editors, Handbook of microalgal culture: applied phycology and 
biotechnology. John Wiley y Sons, Ltd. GBR. p. 123-133.

Jad-Allah, K. 2012. Development of cheap and simple culture medium for the microalgae Nannochloropsis sp. based on agricultural grade fertlilizers available in the local market of Gaza Strip (Palestine). J. Al Azhar University Gaza (Natural Sci.) 14:61-76.

Krause, G.H., and E. Weis. 1984. Chlorophyll fluorescence as a tool in plant physiology. 2. Interpretation of fluorescence signals. Photosynth Res. 5:139-157.

Lee, Y., W. Chen, H. Shen, D. Han, Y. Li, H. Jones, J. Timlin, and Q. Hu. 2013. Basic culturing and analytical measurement techniques. In: A. Rhichmond, and Q. $\mathrm{Hu}$, editors, Handbook of microalgal culture: applied phycology and biotechnology. John Wiley y Sons, Ltd. GBR. p. 37-68.

Lichenthaler, H. 1987. Chlorophyll and carotenoids: pigments of photosynthetic membranes. Methods Enzymol. 148:350-82.

Liu, J., and Q. Hu. 2013. Chlorella: industrial production of cell mass and chemicals. In: A. Rhichmond, and Q. $\mathrm{Hu}$, editors, Handbook of microalgal culture: applied phycology and biotechnology. John Wiley y Sons, Ltd. GBR. p. 329-338.

Lowry, O., and N. Rosenbrough. 1951. Protein measurement with the folin phenol reagent. J. Biol. Chem. 193:265272.

Masojídek, J., G. Torzillo, and M. Koblízek. 2013. Photosynthesis in microalgae. In: A. Rhichmond, and $\mathrm{Q}$. Hu, editors, Handbook of microalgal culture: applied phycology and biotechnology. John Wiley y Sons, Ltd. GBR. p. 21-36.

Mellis, A., L. Zhang, M. Forestier, M. Ghirardi, and M. Seibert. 2000. Sustained photobiological hydrogen gas production upon reversible inactivation of oxygen evolution in the green alga Chlamydomonas reinhardtii. Plant Physiol. 122:127-136.

Molina, G.E., H. Belarbi, F.G. Acién, A. Robles, and Y. Chisti. 2003. Recovery of microalgal biomass and metabolites: process options and economics. Biotech. Adv. 20:491-515.

Nieves, M., D. Voltolina, M. Sapién, H. Gerhardus, A.L. Robles, and M. Villa. 1996. Culturing microalgae with agricultural fertilizers. Riv. Ital. Acquacoltura 3:81-84.

Norsker, N.H., M.J. Barbosa, M. Vermue and R.H. Wijffels. 2011. Microalgal production - A close look at the economics. Biotechnol. Adv. 29:24-27.

Ortiz, M., C. Cortés, J. Sánchez, J. Padilla, y A.M. Otero. 2011. Evaluación del crecimiento de la microalga
Chlorella sorokiniana en diferentes medios de cultivo en condiciones autotróficas y mixotróficas. ORINOQUIA 16:11-19.

Piña, P., A. Medina, M. Nieves, S. Leal, J.A. López, y M. Guerrero. 2007. Cultivo de cuatro especies de microalgas con diferentes fertilizantes utilizados en acuicultura. Rev. Invest. Mar. 28:225-236.

Richmond, A. 1999. Physiological principles and modes of cultivation in mass production of photoautotrophic microalgae. In: Z. Cohen, editor, Chemicals from microalgae. T.J. International Ltd, Padstow, GRB. p. 353-386.

Rocha, J.M.S., J.E. García, and M.H. Henriques. 2003. Growth aspects of the marine microalga Nannochloropsis gaditana. Biomol. Eng. 20:237-242.

Sánchez, H., J. Juscamaita, J. Vargas, y R. Oliveros. 2008. Producción de la microalga Nannochloropsis oculata (Droop) Hibberd en medios enriquecidos con ensilado biológico de pescado. Ecol. Apl. 7:149-158.

Schenk, P., A. Thomas-Hall, S. Stephens, U. Marx, J. Mussgnung, C. Posten, O. Kruse, and B. Hankamer. 2008. Second generation biofuels: high-efficiency microalgae for biodiesel production. BioEnergy Res. 1:20-43.

Schubert, E. 2003. Nonmotile coccoid and colonial green algae. In: J.D. Wehr, and R. Sheath, editors, Freshwater algae of North America: ecology and classification. CA, USA. p. 253-307

Silva, A.M., and G. Torzillo. 2012. Nitrogen and phosphorus removals by means of laboratory batch cultures of the microalga Chlorella vulgaris and the cyanobacterium Planktothrix isothrix grown as monoalgal and as cocultures. J. Appl. Phycol. 24:267-276.

Silva, A.M., G. Torzillo, J. Kopecky, and J. Masojıdek. 2013. Productivity and biochemical composition of Phaeodactylum tricornutum (Bacillariophyceae) cultures grown outdoors in tubular photobioreactors and open ponds. Biomas. Bioenergy 54:115-122.

Simental, J.A, and M. Sánchez. 2003. The effect of agricultural fertilizer on growth rate of benthic diatoms. Aquacult. Eng. 27:265-272.

Simental, J.A., M.P. Sánchez, and J. Correa. 2001. Biochemical composition of benthic marine diatoms using as culture medium a common agricultural fertilizer. J. Shellfish Res. 20:611-617.

Tam, N., and Y.S. Wong. 1990. The comparison of growth and nutrient removal efficiency of Chlorella pyrenoidosa in settled and activated sewages. Environ. Pollut. 65:93-108

Agron. Mesoam. 27(2):265-275. 2016 ISSN 2215-3608 
Tam, N., and Y.S. Wong. 1996. Effect of ammonia concentrations on growth of Chlorella vulgaris and nitrogen removal from media. Bioresour. Technol. 57:45-50.

Tredici, M. 2010. Photobiology of microalgae mass cultures: understanding the tools for the next green revolution. Biofuels 1:143-162.

Torzillo, G., P. Accolla, E. Pinzani, and J. Masojidek. 1996. In situ monitoring of chlorophyll fluorescence to assess the synergistic effect of low temperature and high irradiance stress in Spirulina cultures grown outdoors in photobioreactors. J. Appl. Phycol. 7:1-9.

Torzillo, G., C. Faraloni, M. Silva, J. Kpecky, and J. Masojidek. 2012. Photoacclimation of P. tricornutum (Bacillariophycea) cultures grown outdoors in photobioreactors and open ponds. Eur. J. Phycol. 47: 169-181.

Torzillo, G., and A. Vonshak. 2013. Environmental stress physiology with references to mass cultures. In: A. Richmond, editor, Handbook of microalgal mass cultures. Blackwell Science, River Street, USA. p. 90-113.

Valenzuela, E., F. Lafarga De La Cruz, R. Milán, y F. Núñez. 2005. Crecimiento, consumo de nutrientes y composición proximal de Rhodomonas sp. cultivada en medio F/2 y fertilizantes agrícolas. Cienc. Mar. 31:79-89.

Watanabe, M. 2005. Freshwater cultura media. In: R. Andersen, editor, Algal culturing techniques. Elsevier Academic Press, California, USA. p. 13-20. 
